\title{
Personality Predicts the Brain's Response to Viewing Appetizing Foods: The Neural Basis of a Risk Factor for Overeating
}

\author{
Luca Passamonti, ${ }^{1,2}$ James B. Rowe, ${ }^{1,3,5}$ Christian Schwarzbauer, ${ }^{1,4}$ Michael P. Ewbank, ${ }^{1}$ Elisabeth von dem Hagen, ${ }^{1}$ and \\ Andrew J. Calder ${ }^{1}$ \\ ${ }^{1}$ Cognition and Brain Sciences Unit, Medical Research Council, Cambridge CB2 7EF, United Kingdom, ${ }^{2}$ Istituto di Scienze Neurologiche, Consiglio \\ Nazionale delle Ricerche, 87050 Piano Lago di Mangone (Cosenza), Italy, Departments of ${ }^{3}$ Clinical Neurosciences and ${ }^{4}$ Psychiatry, University of Cambridge, \\ Cambridge CB2 2QQ, United Kingdom, and ${ }^{5}$ Behavioural and Clinical Neuroscience Institute, University of Cambridge, Cambridge CB2 3EB, United \\ Kingdom
}

Eating is not only triggered by hunger but also by the sight of foods. Viewing appetizing foods alone can induce food craving and eating, although there is considerable variation in this "external food sensitivity" (EFS). Because increased EFS is associated with overeating, identifying its neural correlates is important for understanding the current epidemic of obesity. Animal research has identified the ventral striatum, amygdala, hypothalamus, medial prefrontal and premotor cortices as key interacting structures for feeding. However, it is unclear whether a similar network exists in humans and how it is affected by EFS. Using functional magnetic resonance imaging, we showed that viewing appetizing compared with bland foods produced changes in connectivity among the human ventral striatum, amygdala, anterior cingulate and premotor cortex that were strongly correlated with EFS. Differences in the dynamic interactions within the human appetitive network in response to pictures of appetizing foods may determine an individual's risk of obesity.

Key words: connectivity; fMRI; food; emotion; amygdala; striatum; cingulate cortex

\section{Introduction}

Eating behavior can be triggered by internal signals of energy deficits such as hunger. However, external cues such as the sight of appetizing foods can also evoke a desire to eat, even in the absence of hunger. This external food sensitivity (EFS) varies considerably across individuals, and high EFS has been associated with increased risk for obesity when exposed to a food-rich environment (Braet and Van Strien, 1997; Hörchner et al., 2002; Braet et al., 2007; Burton et al., 2007; Elfhag et al., 2007, 2008). Given recent concerns that advertisements and packaging can initiate and maintain increased food intake, identifying the neurobiological basis of EFS is crucial for understanding obesity.

Animal research has implicated a broad network mediating feeding behavior, including subcortical (ventral striatum, amygdala, hypothalamus), allocortical (hippocampus), and frontal cortical regions (motor, premotor, orbital and medial prefrontal cortices) (Masuda et al., 1997; Nishijo et al., 1997; Baldo et al.,

Received 0ct. 15, 2008; revised Nov. 14, 2008; accepted Nov. 16, 2008.

This work was supported by the Medical Research Council under project code U.1055.02.001.00001.01 (A.J.C.). James B. Rowe is supported by the Wellcome Trust. We thank Andrew Lawrence for helpful discussions, Giuseppina Morganti for helping with the reference section, Simon Strangeways for helping in the preparation of the figures, and Cognition and Brain Sciences Unit radiographers for their invaluable support in data acquisition.

Correspondence should be addressed to either of the following: Dr. Luca Passamonti, Istituto di Scienze Neurologiche, Consiglio Nazionale delle Ricerche, 87050 Piano Lago di Mangone (Cosenza), Italy, E-mail: I.passamonti@isn.cnr.it; or Dr. Andrew J. Calder, Cognition and Brain Sciences Unit, Medical Research Council, 15 Chaucer Road, Cambridge CB2 7EF, UK, E-mail: andy.calder@mrc-cbu.cam.ac.uk.

DOI:10.1523/JNEUROSCI.4966-08.2009

Copyright $\odot 2009$ Society for Neuroscience $\quad$ 0270-6474/09/290043-09\$15.00/0
2005; Kelley et al., 2005). Functional imaging research in humans has also highlighted the role of these regions in food processing (Small, 2002; Killgore et al., 2003; DelParigi et al., 2007). However, critical questions remained unanswered.

Previous demonstrations of independent regional effects do not address potentially important interactions that occur in the neural network implicated in processing foods. The change in the blood oxygenation level-dependent (BOLD) response in any brain region is the result of inputs from multiple brain sources and intracortical processing in that area (Logothetis et al., 2001; Goense and Logothetis, 2008). In contrast, connectivity analysis examines the interaction between pairs of specific brain regions. Here, we used psychophysiological interaction (PPI) to address how the physiological connectivity (coupling) between pairs of regions is affected by psychological context (e.g., viewing appetizing vs bland foods) (Friston et al., 1997). Of particular relevance, recent work in rats examining the correlation between brain regions expressing "immediate early gene and proenkephalin" (measures of neuronal metabolism) indicates that the connectivity between the amygdala, ventral striatum (nucleus accumbens core) and prefrontal cortex is modulated when animals are exposed to contextual cues associated with appetizing food (Schiltz et al., 2007). These regions are directly anatomically interconnected (Kunishio and Haber, 1994; Friedman et al., 2002; Haber et al., 2006; Barbas, 2007; Ghashghaei et al., 2007). Hence, a formal analysis of their connectivity in humans in response to viewing foods, and the modulation of this connectivity by the EFS 
personality trait, could provide new insights into the neural basis of vulnerability to obesity.

We investigated these issues using functional magnetic resonance imaging (fMRI) to focus on the neural correlates of viewing appetizing compared with bland foods in healthy volunteers. Our main hypothesis was that the connectivity in the feeding network identified by animal research would be regulated by the interactive effects of viewing appetizing (relative to bland) foods and individual variability in EFS. To directly test this hypothesis, we studied PPI using general linear models (GLMs) of fMRI data.

\section{Subjects and Methods}

Subjects. Twenty-one right-handed healthy volunteers with normal or corrected to normal vision completed the study for payment (10 females; age range, 19-39; mean age, 25.3 years; mean intelligence quotient, 119.8; SD, 21.6; mean body mass index, 24; SD, 4.6). Participants were nonsmokers with no neurological or psychiatric history, including eating disorders. The study was approved by the local ethics committee, and subjects provided written informed consent. Subjects fasted for at least $2 \mathrm{~h}$ before the fMRI session and completed the Dutch Eating Behavior Questionnaire (DEBQ) which assesses EFS (van Strien et al., 1986). The DEBQ also assesses two additional factors implicated in obesity that are not specifically related to external food cues: overeating in response to emotional distress (DEBQ-emotional) and the tendency to suddenly abandon an intense dieting regime (DEBQrestrained) (van Strien et al., 1986). In addition, participants completed the Spielberger State and Trait Anxiety Inventory (Spielberger et al., 1983) and the Center for Epidemiological Studies Depression Scale (CES-D scale) (Sawyer-Radloff, 1977) to exclude any confounding effect by other personality factors associated with overeating as a result of emotional distress. Before and after scanning, subjects also completed the "hunger questionnaire," a self-report measure of subjective hunger (Friedman et al., 1999).

fMRI task. During scanning, subjects viewed full-color photographs of foods consistently identified as highly appetizing (e.g., chocolate cake, ice cream) or bland (e.g., rice, potatoes) (Fig. 1). There were 30 exemplars of each class. Stimuli were selected from ratings made by a different group of individuals $(n=12)$. Ratings were made on a Likert scale (from 1 to 7 ) and indicated the degree to which each stimulus was pleasant, appetizing, and arousing. Mean ratings for the stimuli used are shown in supplemental Table 1, available at www.jneurosci.org as supplemental material. Wilcoxon's signed rank tests showed that appetizing foods were rated as significantly more pleasant and appetizing than bland foods $(p<0.005)$. Stimulus categories did not differ significantly on ratings of arousal $(p>$ 0.05). All photographs were presented via an angled mirror above the subjects' eyes, which reflected back-projected images from a translucent screen in the bore of the magnet behind the subject's head. Stimuli were presented in alternating $21 \mathrm{~s}$ epochs each containing six food images from one category (either appetizing or bland) intermixed with six null events. A total of 18 epochs of each category were presented. The transition between the $21 \mathrm{~s}$ epochs was not signaled, each food stimulus was repeated on average 3.5 times, and the identity of the repeated images was counterbalanced across subjects.

Each "food trial" comprised a $1000 \mathrm{~ms}$ presentation of a photograph (either appetizing or bland food) followed by a low contrast central cross (750 ms). The food photographs subtended horizontal and vertical visual angles of $\sim 11$ and $10^{\circ}$, respectively. Each image was displaced by $\sim 0.8$
18 'Bland' epochs:

(21 sec. each):

6 food trials and 6 null events

in pseudorandom order
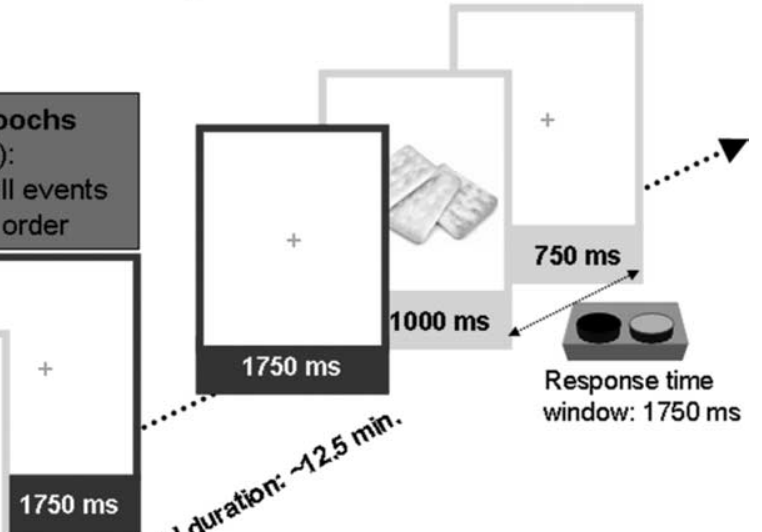

$750 \mathrm{~ms}$

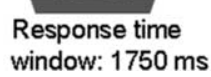

window: $1750 \mathrm{~ms}$
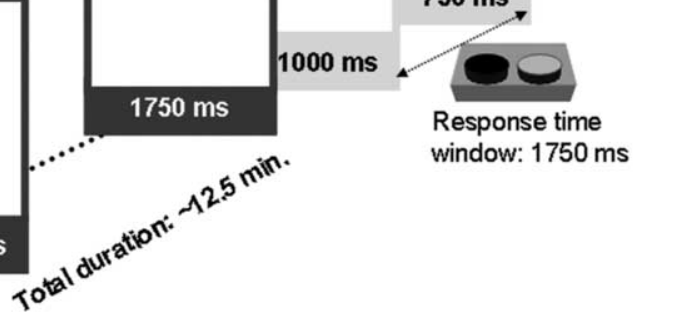

Figure 1. fMRI paradigm and examples of stimuli used. Subjects were shown alternating $21 \mathrm{~s}$ epochs containing pictures of either appetizing or bland foods (18 epochs of each). Each epoch comprised six food trials interspersed with six null events. See Materials and Methods for a full description of the paradigm.

degrees to the left or right of center, and subjects were asked to indicate its position by making a two-choice (left/right) button response within the $1750 \mathrm{~ms}$ trial duration. Null events constituted a $1750 \mathrm{~ms}$ presentation of the same low contrast central cross. The stimuli during each epoch were pseudorandomized with respect to trial type (food trial or null event), such that no more than three consecutive trials were of the same format (food trial or null event). Pseudorandomization enhanced design efficiency while preserving the unpredictability of stimulus onsets in naive subjects. The total experiment duration was $12 \mathrm{~min}$ and $36 \mathrm{~s}$.

Image acquisition and preprocessing. MRI scanning was performed on a 3-Tesla Tim Trio (Siemens) with a head coil gradient set at the Medical Research Council, Cognition and Brain Sciences Unit. Whole-brain data were acquired with echo-planar $2^{*}$-weighted imaging (EPI), sensitive to the BOLD signal contrast ( 46 coronal slices, $3 \mathrm{~mm}$ thickness; repetition time, $2800 \mathrm{~ms}$; echo time, $30 \mathrm{~ms}$; flip angle, $78^{\circ}$; field of view, $192 \mathrm{~mm}$; voxel size, $3 \times 3 \times 3 \mathrm{~mm}$ ). The first three volumes were discarded to allow for equilibration effects. T1-weighted structural images were acquired at a resolution of $1 \times 1 \times 1 \mathrm{~mm}$. Data were analyzed using statistical parametric mapping 5 (SPM5) software (www.fil.ion. ucl.ac.uk/spm/). The EPI images were since interpolated in time to correct for slice time differences and realigned to the first scan by rigid body transformations to correct for head movements. The mean EPI was computed for each subject and inspected to ensure that none showed excessive signal dropout in medial temporal and ventral anterior cingulate cortices (ACCs). EPI and structural images were coregistered and normalized to the standard template in MNI space (Montreal Neurological Institute-International Consortium for Brain Mapping) using linear and nonlinear transformations and smoothed with a Gaussian kernel of fullwidth at half-maximum $8 \mathrm{~mm}$.

Analysis of regional effects. This analysis was used to functionally define the MNI reference coordinates for the ventral striatum and amygdala which were used as "source" regions for the PPI connectivity analyses. Another aim was to verify that the viewing appetizing relative to bland foods elicits the previously reported food processing neural network (Small, 2002; Killgore et al., 2003; DelParigi et al., 2007).

A random effects model was implemented using a two-stage process (first and second level).This random-effects analysis allows inferences about the general population from which subjects were drawn. For each 
Table 1. Main effect of appetizing versus bland food presentation ( $n=21$, one-sample $t$ test on regional BOLD response)

\begin{tabular}{|c|c|c|c|c|c|}
\hline \multirow[b]{2}{*}{ Cerebral region } & \multirow[b]{2}{*}{ Side } & \multirow[b]{2}{*}{ tscore } & \multicolumn{3}{|c|}{ MNI coordinates } \\
\hline & & & $x$ & $y$ & $z$ \\
\hline \multirow[t]{2}{*}{$\operatorname{vACC}^{a}$} & $\mathrm{~L}$ & $7.02^{*}$ & -12 & 36 & -6 \\
\hline & $\mathrm{R}$ & $6.88^{* *}$ & 14 & 40 & 0 \\
\hline \multirow[t]{2}{*}{ Dorsolateral prefrontal cortex } & $\mathrm{L}$ & 5.14 & -22 & 26 & 34 \\
\hline & $\mathrm{R}$ & 5.37 & 20 & 36 & 40 \\
\hline Frontal pole & $\mathrm{L}$ & 5.57 & -6 & 66 & -8 \\
\hline \multirow[t]{2}{*}{ Middle temporal gyrus } & $\mathrm{L}$ & 4.70 & -52 & -10 & -16 \\
\hline & $\mathrm{R}$ & 4.57 & 56 & -2 & -22 \\
\hline Superior temporal gyrus & $\mathrm{R}$ & 4.29 & 56 & -16 & 0 \\
\hline Posterior cingulate cortex & - & 4.47 & 0 & -42 & 34 \\
\hline Ventral striatum & $\mathrm{R}$ & $5.81^{*}$ & 6 & 6 & -4 \\
\hline Amygdala & $\mathrm{L}$ & $5.10^{*}$ & -30 & 2 & -20 \\
\hline \multirow[t]{2}{*}{ Extrastriate visual cortex ${ }^{a}$} & $\mathrm{~L}$ & 6.99 & -30 & 62 & -12 \\
\hline & $\mathrm{R}$ & 6.94 & 36 & -48 & -6 \\
\hline
\end{tabular}

All regions in the whole-brain analysis met the criteria of $p<0.001$, uncorrected. ROls met the criteria of ${ }^{*} p<0.001$ or ${ }^{* *} p<0.005$, FWE, svc. L, Left; R, right. ${ }^{a}$ Regions also met the threshold of $p<0.05$ (FWE), whole-brain correction.

subject we used a GLM to assess regionally specific effects of task parameters on BOLD indices of activation (Friston et al., 1994). The model included two experimental factors (appetizing and bland food presentation), effects of no interest (null events), and the realignment parameters to account for residual motion-related variance. Low-frequency signal drift was removed using a high-pass filter (cutoff $128 \mathrm{~s}$ ) and autoregressive(1) modeling of temporal autocorrelations was applied.

All events (appetizing, bland, and null) were modeled by $\delta$ functions convolved by the hemodynamic response function (HRF). The $\beta$-weight derived from the GLM therefore reflects the average response to all events of a given type. Because we used the canonical HRF, the mean peak activation after an event is directly proportional to the $\beta$-weight. Null events acted as a baseline and enhanced food stimulus related variance in regional BOLD signal. This increases the power of subsequent connectivity analysis. From the first-level analyses, we generated contrast images for appetizing versus bland foods. These are contrast images (of the voxel-wise differences in $\beta$-estimates for appetizing and bland foods) but not statistical images. The second-level analysis of the group used these contrast images in a new GLM from which we generated statistical images, SPM $\{t\}$ maps. With balanced designs at the first level (i.e., similar events for each subject, in similar numbers), this second-level analysis closely approximates a true mixed effects design, with both within and between subjects variance.

The 21 contrast images from individual subjects' first-level analyses were entered into second-level models to explore the main effect of the appetizing versus bland contrast in the whole group (one sample $t$ test) and to identify any effect of the psychometric measures (any DEBQ, anxiety, and depression score) on the isolated brain responses for the same contrast (regression models).

The first-level (single subject) data were not statistically thresholded before inclusion in the second level. Two approaches to threshold second-level maps were applied. First, for small volume corrections (svc) regarding previous hypotheses in specific regions of interest (ROIs), the threshold was set at $p<0.05$ family-wise error (FWE) (Worsley et al., 1996; Friston, 1997). Because brain regions might be very different in size (in particular subcortical compared with cortical areas), we treated them as separate hypotheses and adjust the significance for multiple hypotheses testing using Dunn-Sidak correction for multiple tests (Howell, 2007). The amygdala, striatum, premotor, and ACC were defined as a priori ROIs based on the animal and human literature showing their key role in feeding behavior and in processing reward stimuli such as foods (Rolls et al., 1980; LaBar et al., 2001; Ehrsson et al., 2003; Killgore et al., 2003; Dresel et al., 2005; Haber et al., 2006; Killgore and Yurgelun-Todd, 2006; Alonso-Alonso and Pascual-Leone, 2007; Berthoud, 2007; Cornier et al., 2007; DelParigi et al., 2007; Schiltz et al., 2007; Berthoud and Morrison, 2008). All ROIs were defined using the Marsbar software incorporating the "aal.02" atlas for automatic anatomical labeling (http:// marsbar.sourceforge.net/) (Tzourio-Mazoyer et al., 2002). Second, we report other regions which were not predicted but met a threshold of $p<0.001$, uncorrected, 10 contiguous voxels as defined functionally, by their suprathreshold $t$ values.

Connectivity analyses: PPI in a GLM. The physiological connectivity between two brain regions can vary with the psychological context (Friston et al., 1997). Here, we were interested in connectivity that is modulated by the context of viewing appetizing versus bland foods. This constitutes a PPI (Friston et al., 1997). We sought to identify "target" regions that showed differential connectivity according to the context (appetizing vs bland) with each of two source regions (the ventral striatum and the amygdala) consistently implicated in feeding and food reward by animal and human research (Rolls et al., 1980; Nishijo et al., 1988; Masuda et al., 1997; Baldo et al., 2005; Balleine and Killcross, 2006; O'Doherty et al., 2006); the amygdala and ventral striatum have also been implicated in other types of rewards such as money (Knutson and Bossaerts, 2007) and positive feedback (Cools et al., 2002). We also explored any connectivity pattern arising from the ventral ACC, given its wider role in emotion regulation (Davidson et al., 2000; Ochsner and Gross, 2005; Delgado et al., 2008) and extinction (Phelps et al., 2004; Delgado et al., 2008) (supplemental material, available at www. jneurosci.org). The ventral ACC was identified using the same contrast used to locate the amygdala and ventral striatum source regions.

Using PPI, regions are not identified because their activity is correlated with activation in the source region or the presence/absence of appetizing foods but rather because of the interaction between these two variables. More specifically, we sought to identify target regions for which the change in connectivity with the source region varied as a function of EFS. We refer to this latter analysis examining the interaction with ESF as a "higher-order PPI."

Separate connectivity analyses (PPIs) were carried out, using the ventral striatum, amygdala, and ventral ACC regions from the whole-group analysis as the sources in each (supplemental Figs. 1, 2, available at www.jneurosci.org as supplemental material). The PPI analyses used the same basic procedure, so it is described for the ventral striatum PPI alone. For each subject, we computed the appetizing versus bland food contrast to determine the local maximum that was the nearest voxel to the activation peak in the ventral striatum defined by the whole-group activation clusters (Table 1). Next, $10 \mathrm{~mm}$ spheres were constructed around the subject-specific local maximum and the time series for each subject computed using the first eigenvariate from all voxels' time series. Using this approach, the center of the ventral striatum for each subject was the most significant voxel. This meant that the centers of the spheres were slightly different across subjects; thus, we also used a standardized approach using the same $10 \mathrm{~mm}$ spheres for all subjects (centers for all subjects in the ventral striatum: MNI, $x=6, y=6, z=-4$; in the amygdala: MNI, $x=-30, y=2, z=-20$; and in the ventral ACC: MNI, $x=-12, y=36$, $z=-6$ which are all the maximal voxels for the appetizing vs bland food contrast) (Table 1). Regardless of the approach used to extract the time series in the source regions, we obtained highly consistent results [supplemental Results (available at www.jneurosci.org as supplemental material), Analysis of connectivity: PPIs using the same center of the sphere in the source regions for all subjects].

The BOLD time series for each subject was deconvolved to estimate a "neuronal time series" for the source region, using the PPIdeconvolution parameter defaults in SPM5 (Gitelman et al., 2003). The psychophysiological interaction term (PPI regressor) was calculated as the element-by-element product of the ventral striatum neuronal time series and a vector coding for the main effect of task ( 1 for appetizing foods, -1 for bland foods, and 0 for null events). This product was reconvolved by the canonical HRF. The model also included the main effects of task convolved by the HRF, the neuronal time series for each source, and the movement regressors as effects of no interest. Subject- 
specific PPI models were run, and contrast images were generated for positive and negative PPIs. The identified regions had greater or lesser coupling with the source regions according to the context of appetizing versus bland food presentation.

The 21 first-level contrast images were then entered into second-level GLM analyses for the contrast of interest (appetizing vs bland), and SPM $\{t\}$ maps were generated using Gaussian Random Field theory to make statistical inferences (Friston et al., 1994). The following contrasts were assessed: (1) brain regions that showed changes in connectivity with the source regions for the appetizing versus bland foods context (regardless of variations in any psychometric score) (one sample $t$ test); (2) regions for which the change in connectivity with the source region (after appetizing vs bland food context) were correlated with the individual variation in external food sensitivity (regression model). Correlations with other scales from the DEBQ (DEBQ-emotional, and DEBQrestrain) and with anxiety and depression scores were also investigated (regression models). The second-level models also all included subjects' change in hunger across the experiment (i.e., difference between selfassessed hunger score before and after the scanning session) as a covariate of no interest to remove possible confounding effects of this variable. The same two approaches reported before were used to statistically threshold maps.

\section{Results}

\section{Subjects}

Subjects' scores on the EFS and other DEBQ scales were as follows: external food sensitivity (DEBQ-external): range 23-47, mean $=34.4, \mathrm{SD}=6.3$; DEBQ-emotional: range $16-53$, mean $=$ $34, \mathrm{SD}=11.2$; DEBQ-restrain, range $10-42$, mean $=23.3, \mathrm{SD}=$ 9.7. Scores on the Hunger Questionnaire before (and after) scanning were as follows: range 9 (16) to 34 (43), mean 24.1 (29.9), $\mathrm{SD}=6.8$ (7.16). Subjects' scores on other personality dimensions were as follows: trait anxiety: range $21-52$, mean $=36.52, \mathrm{SD}=$ 8.39; state anxiety: range $20-43$, mean $=30.71, \mathrm{SD}=6.76$; depression $($ CES-D): range $1-24$, mean $=11, \mathrm{SD}=6.20$.

EFS was positively correlated with the change in participants' hunger scores (hunger score after scanning minus hunger score before) ( $r=0.39 ; p=0.03$; one-tailed), but critically showed no correlation with levels of hunger before scanning $(p>0.3)$. Thus, people who were more sensitive to external food-related cues (high EFS) presented a greater increase in their subjective level of hunger after viewing pictures of appetizing foods. Because EFS can operate in the absence of "internal" homeostatic signals of hunger (van Strien et al., 1986; Burton et al., 2007), we excluded any contribution of "change-in-hunger," by including this as a covariate of no interest in the PPI analyses (see Materials and Methods). Remarkably similar results to those reported were found even when the change-in-hunger covariate was not included (supplemental Results, available at www.jneurosci.org as supplemental material); thus, it cannot account for the effects of EFS we observed. There were also no significant correlations between EFS and any other DEBQ or personality measure, nor between the EFS and the body mass index (BMI) $r$ 's $<0.09$, p's $>$ 0.34 . Hence, the effects of EFS cannot be attributed to these other variables.

\section{fMRI behaviorals}

Task accuracy to indicate the position of each food picture in the visual display was high for both appetizing (mean accuracy, 96.7\%; SD, 2.6) and bland (mean accuracy, 94.8\%; SD, 3.1) food presentation $\left(t_{(20)}=1.02 ; p=0.31\right)$, with no significant correlations with EFS or other DEBQ or personality scales, hunger scores or BMI ( $r$ 's $<0.07$; $p$ 's $>0.38)$.

\section{Analysis of regional effects}

The whole-group analysis (regardless of individual differences in any personality measure) identified a group of regions showing an increased BOLD response to appetizing compared with bland foods that included the ventral striatum, the amygdala, and the ventral ACC (Table 1; supplemental Figs. 1, 2, available at www. jneurosci.org as supplemental material). Moreover, the task evoked activations in brain regions previously implicated in food processing (Small, 2002; Killgore et al., 2003; DelParigi et al., 2007).

When we assessed any significant modulation by either the EFS or by any other DEBQ or personality scale (DEBQemotional, DEBQ-restrain, trait/state anxiety, depression) on the isolated brain BOLD responses for the same contrast (appetizing vs bland foods), none of the ROIs met the criteria for statistical significance. The only region outside our a priori ROIs that showed a positive correlation with the external food sensitivity was the anterior superior temporal sulcus $(x=56, y=4, z=$ $-18 ; t=4.57 ; p<0.001$, uncorrected; $r=0.72 ; p<0.001)$.

\section{Analysis of connectivity: PPIs across subjects regardless of individual differences in EFS or other personality measures} In the same way that interactions and main effects are statistically distinct, the interactive influence of EFS on any change in connectivity from viewing appetizing relative to bland foods is also distinct from any overall effect regardless of EFS. First we examined the latter PPIs. The PPIs showing a change in connectivity with the ventral striatum (source region) across all subjects demonstrated no statistically significant effects in our a priori ROIs. However, the whole-brain analysis showed that the frontal pole $(x=-32, y=46, z=10 ; t=4.37 ; p<0.0001$, uncorrected $)$ and the posterior parietal cortex $(x=-46, y=-54, z=44 ; t=4.74$; $p<0.0001$, uncorrected) showed changes in connectivity with the ventral striatum as a function of the psychological context (appetizing vs bland food). No statistically significant PPIs in our a priori ROIs were found in the whole-group analysis (i.e., regardless of any personality measures) using the amygdala as the source region (even at $p<0.01$, uncorrected). Furthermore, no additional regions outside these ROIs met the criteria for statistical significance ( $p<0.001$, uncorrected).

\section{Analysis of connectivity: higher-order PPIs as a function of individual differences in EFS}

For the analysis in which the ventral striatum was the source region, EFS strongly modulated the change in connectivity between the ventral striatum and amygdala, to the sight of appetizing versus bland foods $(x=-26, y=-4, z=-16 ; t=6.33 ; p<$ 0.0001 , FWE svc; $r=0.79 ; p<0.001$ ) (Fig. $2 A$ ), and similarly between the ventral striatum and an area of the premotor cortex involved in the preparation of orofacial movements $(x=56, y=$ $0, z=36 ; t=4.51 ; p<0.05$, FWE svc; $r=0.66$; $p<0.001$ ) (Fig. $2 B$ ) (Ehrsson et al., 2003; Dresel et al., 2005). In both cases, EFS was positively correlated with the change in connectivity ranging from more negative values in low EFS to less negative values in high EFS individuals. A change in connectivity between the ventral striatum and the dorsal ACC was also correlated with EFS $(x=-2, y=28, z=20 ; t=6.01 ; p<0.002$, FWE svc; $r=-0.78$; $p<0.001$ ) (Fig. 2C), but here the correlation was negative (more positive values in low EFS, and less positive values in high EFS).

For the analysis in which the amygdala was the source region, EFS was negatively correlated with the change in connectivity between the amygdala and both the ventral ACC $(x=0, y=26$, $z=-6 ; t=4.54 ; p<0.02$, FWE svc; $r=-0.58 ; p<0.005$ ) (Fig. 


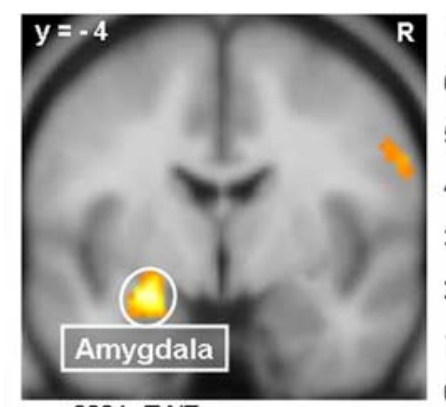

A

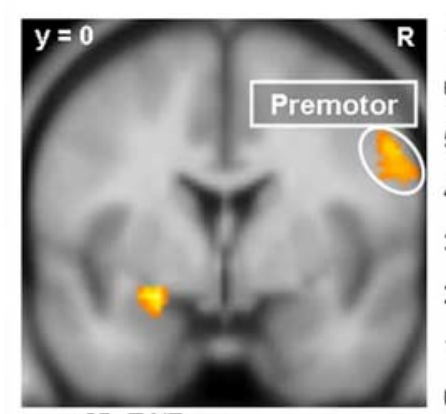

B
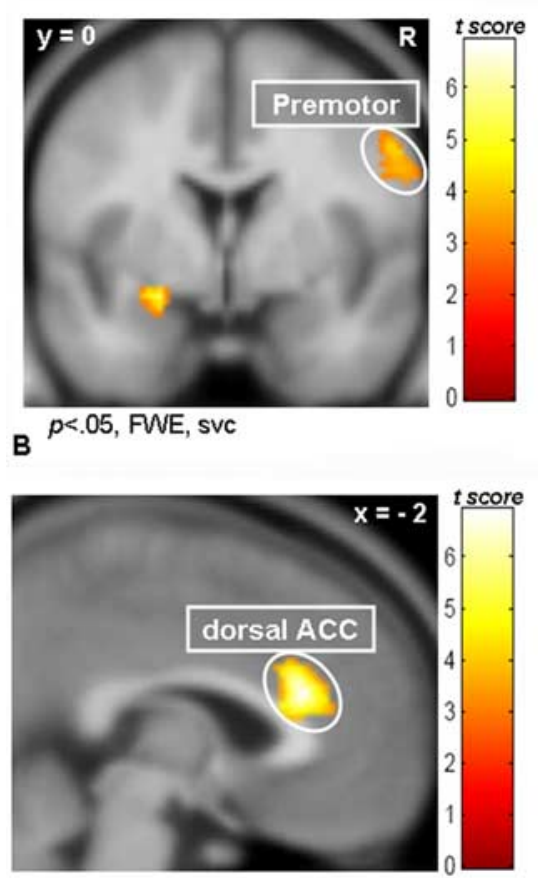

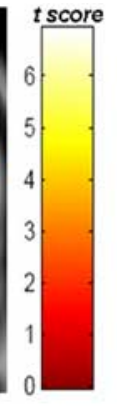

$p<.002$, FWE, sve

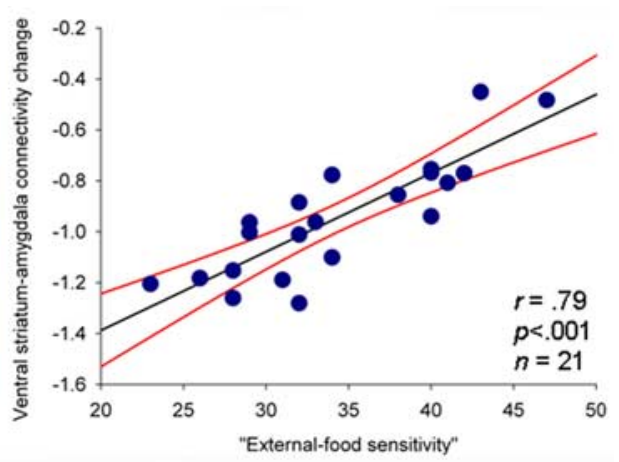

\&
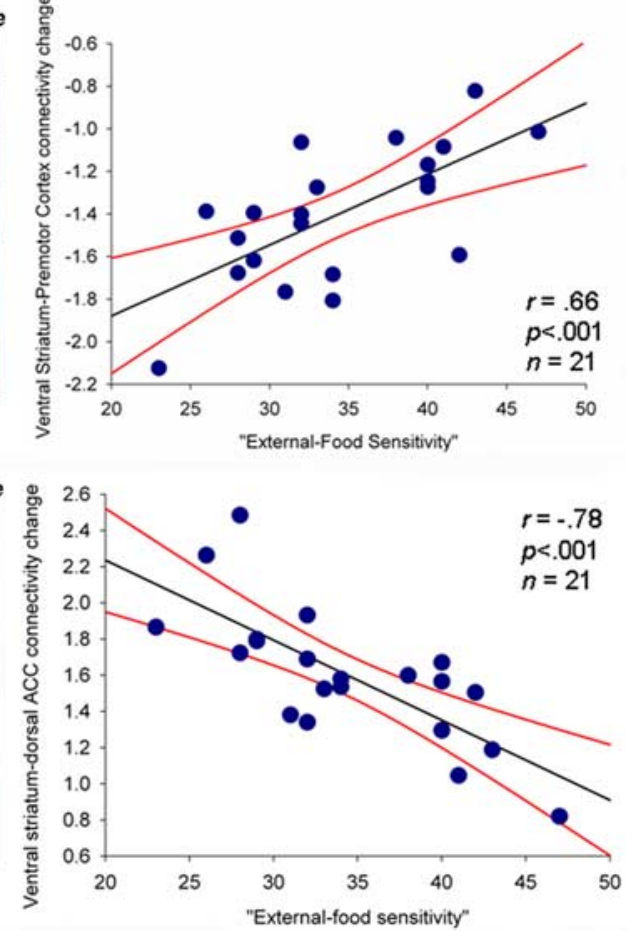

Figure 2. Higher-order PPI from the ventral striatum (source region). $\boldsymbol{A}$, When viewing appetizing versus bland foods, EFS was positively correlated with the change in connectivity between the ventral striatum and the amygdala. $\boldsymbol{B}$, When viewing appetizing versus bland foods, EFS was positively correlated with the change in connectivity between the ventral striatum and the orofacial region of the premotor cortex. C, When viewing appetizing versus bland foods, EFS was negatively correlated with the change in connectivity between the ventral striatum and the dorsal ACC. Color bars represent $t$ statistics. FWE, svc. The $x$ and $y$ coordinates are in MNI space. The regression lines (black) and the $95 \%$ confidence intervals (red lines) are shown. $n$, Number of subjects. Activations are shown at the thresholded $p<0.001$, uncorrected.

$3 A)$ and the dorsal ACC $(x=-2, y=24, z=22 ; t=4.05 ; p<$ 0.05 , FWE svc; $r=-0.59 ; p<0.004$ ) (Fig. $3 B$ ). In both cases, the range of the connectivity changes was from more positive values in low EFS to less positive values in high EFS individuals. We can therefore conclude that in high EFS people there is a reduction in the change in connectivity (regardless of the sign of the changes) between specific "feeding" brain areas when viewing appetizing versus bland food. It is also of interest that the dorsal ACC region identified in this latter analysis using the amygdala as the source, showed a considerable overlap with a similar area identified in the previous analysis using the ventral striatum as the source (Fig. 4). A summary of all higher-order PPIs is shown in Figure 5. A whole-brain analysis showed no other regions with EFSdependent differential connectivity with either the ventral striatum or amygdala ( $p<0.001$, uncorrected).

The PPIs discussed thus far are all indictors of differential connectivity under different contexts. We also examined the average connectivity, pooled over both psychological contexts (i.e., collapsing the appetizing and bland conditions) for each pair of source and target regions identified by higher-order PPIs. As shown in supplemental Figure 4, available at www.jneurosci.org as supplemental material, no correlations were found between the EFS and the average connectivity. In other words, high EFS subjects do not have intrinsically different coupling between the regions of interest (pooled across all food types). This demonstrates that it is only the change in connectivity induced by viewing appetizing versus bland food that is associated with the EFS.

Finally, PPIs arising from the ventral striatum and amygdala were not correlated with the DEBQ-emotional and DEBQ-restrained scales or with other measures of emotional behavior (depression, and state or trait anxiety) (no suprathreshold voxels at $p<0.001$, uncorrected). This highlights the specificity of the EFS in capturing individual differences in the change in brain connectivity related to processing external food cues.

In all reported analyses, source region data from the ventral striatum and amygdala were extracted using subject-specific peak foci (see Subjects and Methods). However, the results were replicated with a different approach, using a standard coordinate for each of the ventral striatum and amygdala for all subjects (supplemental Results, available at www.jneurosci.org as supplemental material).

The fact that connectivity between components of the feeding network (Kelley et al., 2005; Schiltz et al., 2007; Berthoud and Morrison, 2008) were only fully identified by the EFS regression analyses accords with the role of EFS in responsivity to external food cues (van Strien et al., 1986; Burton et al., 2007; Elfhag et al., $2007,2008)$. In other words, the large variance in the change of connectivity is accounted for by individual differences in EFS, with relative decreases as a function of EFS.

\section{Discussion}

We have shown that specific connections in the human brain are differentially affected by EFS, a factor associated with overeating and increased preference for appetizing foods (Braet and Van Strien, 1997; Elfhag et al., 2008), while subjects viewed appetizing compared with bland foods. High EFS was associated with reduced differential connectivity in a network that shows striking similarities with one implicated in feeding behavior by animal research, including the ventral striatum, amygdala, medial prefrontal, and premotor cortices (Kelley et al., 2005).

Work in rats has shown that exposure to external contextual cues associated with highly palatable foods alters connectivity between very similar components of the feeding network (Schiltz et al., 2007). Our current findings demonstrate an analogous 
effect in humans that is critically dependent on EFS. We therefore suggest that individual differences in EFS constitute the behavioral expression of variation in network coupling among neural structures implicated in feeding.

EFS also correlated with subjects' change in hunger before and after viewing pictures of foods. However, it is important to note that the connectivity results were found despite factoring out this change. This accords with the observation that high EFS can affect overeating in the absence of hunger (van Strien et al., 1986), and animal research demonstrating that the network we have identified reflects the rewarding/hedonic aspects of food processing (Berthoud and Morrison, 2008). Consistent with this interpretation, a large sample study has shown that increased EFS is associated with increased preference for the sort of high calorie, sweet foods used in our stimulus set (Elfhag et al., 2008). Here, we suggest that this might operate by affecting connections between components of the "feeding network."

Amygdala-ventral striatum interactions and external food sensitivity

Although PPIs do not indicate the direction of influences, anatomical research demonstrates connections from the amygdala to ventral striatum but not vice versa (Russchen et al., 1985; Friedman et al., 2002). In accord with this, the overeating induced by the stimulation of $\mu$-opioid receptors in the ventral striatum (nucleus accumbens) is blocked by the inactivation of the amygdala, demonstrating that the latter is a critical source of food-related inputs to the ventral striatum (Will et al., 2004; Baldo et al., 2005). Moreover, a recent study in animals showed that the amygdala response to reward-predictive cues precedes that of the ventral striatum (Ambroggi et al., 2008). Hence, connectivity between the amygdala and ventral stria-

tum operates in one specific direction. A reasonable question is why the PPI analyses identified the amygdala from the ventral striatum but not vice versa. There are different possible explanations for this. First, the construction of the PPI terms from ventral striatum and the amygdala might be asymmetrical because of differences in the convolved hemodynamic response functions. Second, the statistical significance of PPIs from the amygdala and the ventral striatum might differ given the different residual error terms in the two regions. However, in accord with anatomical evidence and previous comparative research, it is reasonable to conclude that the pathway from the amygdala to the ventral striatum is a critical route in mediating the individual sensitivity to hedonic aspects of external food cues. as source region.

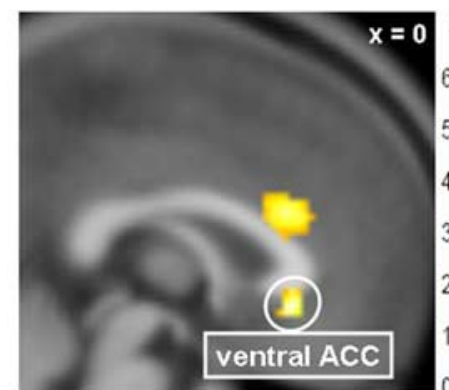

$p<.02$, FWE, svc

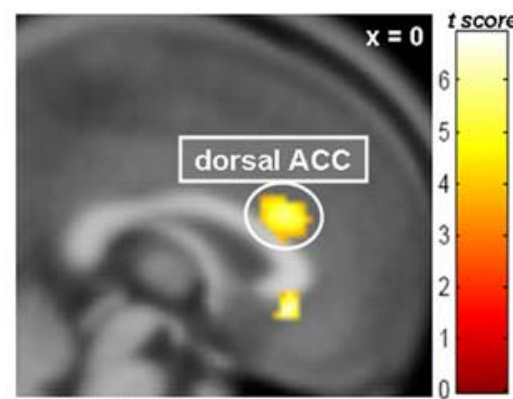

$p<.05$, FWE, svc
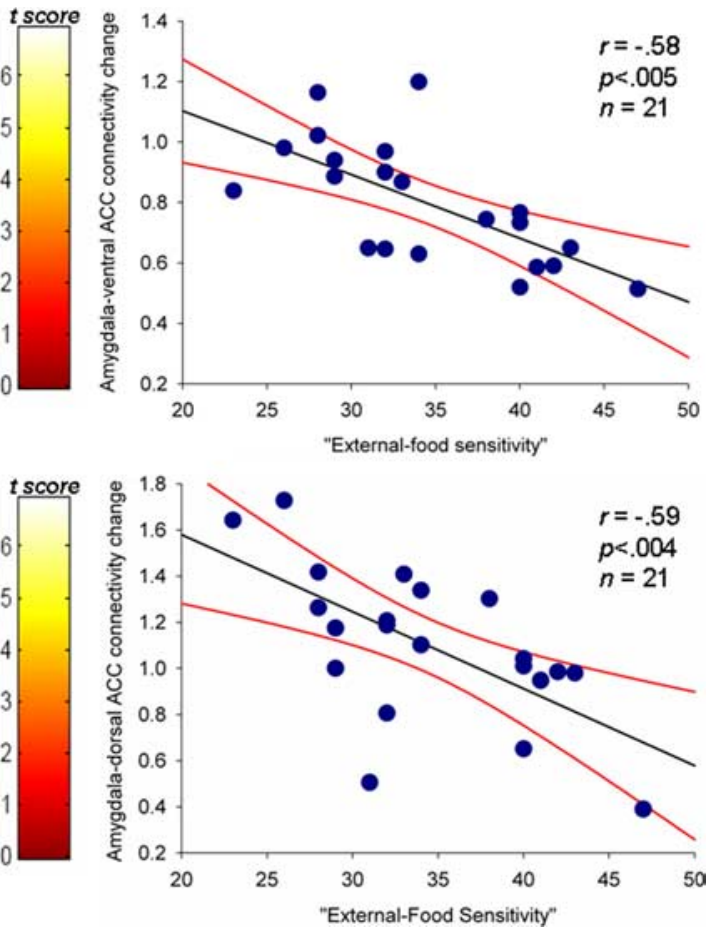

Figure 3. Higher-order PPI from the amygdala (source region; see Results and Subjects and Methods). $\boldsymbol{A}$, When viewing appetizing versus bland foods, EFS was negatively correlated with the change in connectivity between the amygdala and the ventral ACC. $B$, When viewing appetizing versus bland foods, EFS was negatively correlated with the change in connectivity regression lines (black) and the $95 \%$ confidence intervals (red lines) are shown. $n$, Number of subjects. Activations are shown at the thresholded $p<0.001$, uncorrected.
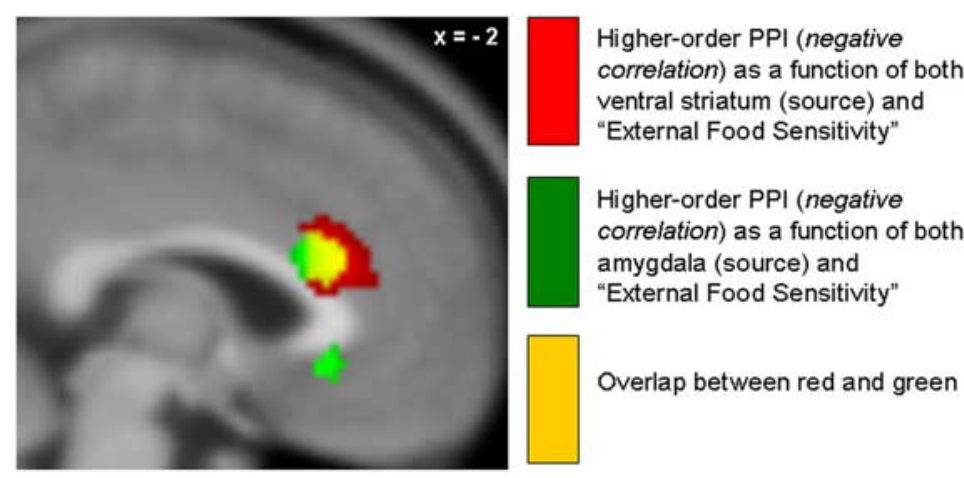

Higher-order PPI (negative correlation) as a function of both amygdala (source) and "External Food Sensitivity"

Overlap between red and green

Figure 4. The dorsal ACC identified in the higher-order PPI analysis (negative correlation) from the ventral striatum (as source region) strongly overlaps with the dorsal ACC identified in the higher-order PPI analysis (negative correlation) from the amygdala

Ventral striatum, amygdala, frontal cortex interactions, and external food sensitivity

The ventral and dorsal anterior cingulate cortex (v/dACC) is strongly anatomically connected with ventral striatum and amygdala (Kunishio and Haber, 1994; Ghashghaei et al., 2007). Researchers in comparative neuroscience have proposed that these connections modulate the response of the ventral striatum and amygdala to appetizing foods and other rewarding stimuli (Haber et al., 2006). The negative correlation between the change in the dACC-ventral striatum connectivity and v/dACC-amygdala connectivity as a function of EFS might therefore represent altered prefrontal-limbic interactions during the processing of hedonic and rewarding aspects of foods (Berthoud, 2007).

Anatomical evidence showing projections from ACC to ven- 


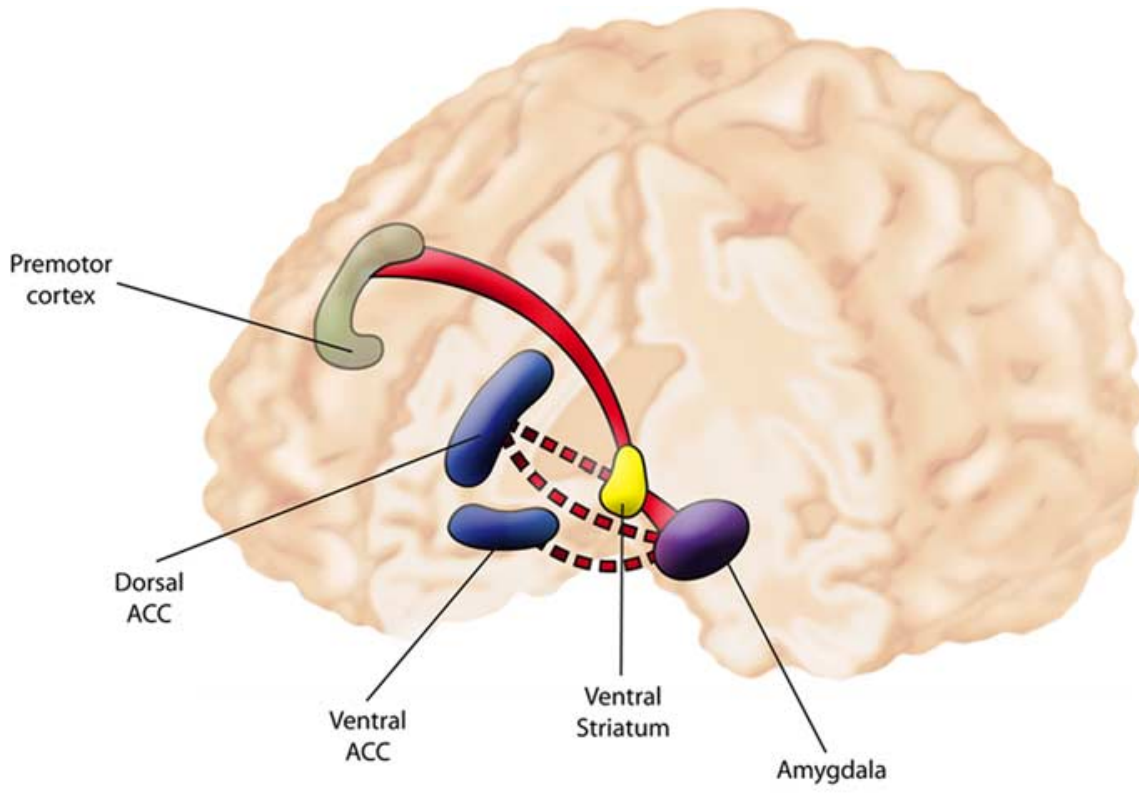

Higher-order PPIs (positive correlations) with "External Food Sensitivity"

ロロロロロ Higher-order PPIs (negative correlations) with "External Food Sensitivity"

Figure 5. Summary of the higher-order PPI from the ventral striatum and the amygdala source regions. EFS was positively correlated with the connectivity between the amygdala and the ventral striatum, attributed to the emotional and motivational states evoked by the sight of appetizing foods (red solid line). A similar correlation was found with the change in connectivity between the ventral striatum and the orofacial region of premotor cortex that might enable the preparation of motor acts necessary for eating. EFS also show negative correlations with the change in connectivity between the dorsal and ventral ACC and the amygdala and between the dorsal ACC and the ventral striatum (red dotted lines), interpreted as altered prefrontal-limbic interactions. Note also that connections shown indicate changes in connectivity as a function of both viewing appetizing versus bland food and of the external food sensitivity but do not necessarily represent anatomical pathways.

tral striatum, but not vice versa (Kunishio and Haber, 1994; Haber and McFarland, 1999; Haber et al., 2006), allow us to infer the directionality of the prefrontal and ventral striatum interactions. However, in the case of connectivity between the v/dACC and the amygdala, anatomical projections are bidirectional (although vACC sends more projections to amygdala than it receives and vice versa for dACC) (Ghashghaei et al., 2007), and we would not wish to exclude potential contributions of connections from the amygdala to prefrontal cortex.

Furthermore, EFS negatively correlated with the change in connectivity between the vACC and a "gustatory" region of the orbitofrontal cortex (Small et al., 2007) (supplemental material, available at www.jneurosci.org), also implicated in coding the rewarding value of food (O'Doherty, 2004). These interactions accord with the ACC's wider role in extinction of negative emotions (Phelps et al., 2004; Delgado et al., 2008), emotion regulation through cognitive strategies (Ochsner et al., 2004), resolution of emotional conflict (Etkin et al., 2006; Egner et al., 2008), and pain modulation (Ploghaus et al., 2003).

It is also of note that prefrontal cortex (PFC) dysfunction has been proposed as a key factor in obesity (Alonso-Alonso and Pascual-Leone, 2007). For example, diffuse hypoperfusion of the PFC has been described in overeating conditions, such as the Kleine-Levine syndrome (Arias et al., 2002), and the hyperphagic behavior observed in patients with degenerative dementia has been shown to positively correlate with the right PFC atrophy (Whitwell et al., 2007).

The PPI using the ventral striatum as source region also identified a change in connectivity with the orofacial regions of premotor cortex which might be explained by the existence of both direct and indirect (via ventral tegmental area) anatomical pathways (Draganski et al., 2008) (see also Joel, 2001). The premotor/motor cortices also form part of animal models of feeding and may mediate the transformation of the desire to eat (generated by the ventral striatum after inputs from the amygdala) into the preparation of acts necessary for eating (Kelley et al., 2005).

An additional point should be noted. Changes in connectivity were identified by the regression analyses with EFS and not the main effects, emphasizing the importance of this variable in accounting for the wide range of between-subject variance. Similarly, a number of previous fMRI studies have shown correlations with relevant personality variables in a priori regions in the absence of main effects (Bishop et al., 2004; Canli, 2004; Beaver et al., 2006).

\section{Implications for obesity research}

In the current study, variation in EFS was not confounded by differences in body mass index and the neurohormonal dysregulation associated with established obesity. However, it is important to emphasize that increased EFS is associated with overeating and, together with genetic, environmental and other personality risk factors, operate to promote obesity. Studying EFS in the absence of obesity unconfounds these variables. To this extent, we adopt an analogous approach to the investigation of genetic risk factors in disease by demonstrating the effect of a personality trait (rather than a single genetic variant) on a key biological pathway implicated in the disorder (Körner et al., 2008; Li and Loos, 2008).

Structural equation modeling of behavioral data has shown that the relationship between EFS and overeating is mediated by food craving (Burton et al., 2007). In addition, converging evidence shows that the craving for food shown by obese individuals shares many similarities with the loss of control and compulsive behavior associated with drug craving displayed by addicted subjects, and the same brain regions (amygdala, striatum, and v/dACC) have been identified for both (Wang et al., 2004a,b, 2006). Similarly, the amygdala and ventral striatum have also been implicated in processing other rewards including money (Knutson and Bossaerts, 2007) or positive feedback (Cools et al., 2002).

Finally, we emphasize that high EFS individuals displayed a reduction in the change of connectivity between specific brain regions of the feeding network. This decreased or "less efficient" connectivity is analogous to the reductions in connectivity between regions implicated in aggression in anger-prone individuals (Passamonti et al., 2008) and to the more extreme finding of absent connectivity in psychiatric disorders of aggression (Coccaro et al., 2007), generalized anxiety (Monk et al., 2008), schizophrenia (Fakra et al., 2008), and antisocial behavior (Marsh et al., 2008). Together, these findings provide evidence that less efficient connectivity among relevant brain regions might represent 
a "neuronal marker" for increased vulnerability to develop abnormal behaviors, including food craving and overeating.

\section{Conclusions}

Our study reveals how people with high EFS show altered brain connectivity to the sight of appetizing versus bland food. EFS correlated with the change in connectivity between brain regions implicated in motivational aspects of food processing and in emotional regulation. Identification of this relationship provides the critical link between studies of personality traits underlying obesity and the neurobiology of feeding. Our results are also particularly pertinent to recent concerns regarding the power of food advertisements to promote food intake and preferences. In addition, they demonstrate the potential value of individual differences in personality to the developing field of "personalized medicine" (Kumar and Ajilore, 2008).

\section{References}

Alonso-Alonso M, Pascual-Leone A (2007) The right brain hypothesis for obesity. Jama 297:1819-1822.

Ambroggi F, Ishikawa A, Fields HL, Nicola SM (2008) Basolateral amygdala neurons facilitate reward-seeking behavior by exciting nucleus accumbens neurons. Neuron 59:648-661.

Arias M, Crespo Iglesias JM, Pérez J, Requena- Caballero I, Sesar-Ignacio A, Peleteiro-Fernández M (2002) [Kleine-Levin syndrome: contribution of brain SPECT in diagnosis]. Rev Neurol 35:531-533.

Baldo BA, Alsene KM, Negron A, Kelley AE (2005) Hyperphagia induced by GABAA receptor-mediated inhibition of the nucleus accumbens shell: dependence on intact neural output from the central amygdaloid region. Behav Neurosci 119:1195-1206.

Balleine BW, Killcross S (2006) Parallel incentive processing: an integrated view of amygdala function. Trends Neurosci 29:272-279.

Barbas H (2007) Flow of information for emotions through temporal and orbitofrontal pathways. J Anat 211:237-249.

Beaver JD, Lawrence AD, van Ditzhuijzen J, Davis MH, Woods A, Calder AJ (2006) Individual differences in reward drive predict neural responses to images of food. J Neurosci 26:5160-5166.

Berthoud HR (2007) Interactions between the "cognitive" and "metabolic" brain in the control of food intake. Physiol Behav 91:486-498.

Berthoud HR, Morrison C (2008) The brain, appetite, and obesity. Annu Rev Psychol 59:55-92.

Bishop SJ, Duncan J, Lawrence AD (2004) State anxiety modulation of the amygdala response to unattended threat-related stimuli. J Neurosci 24:10364-10368.

Braet C, Van Strien T (1997) Assessment of emotional, externally induced and restrained eating behaviour in nine to twelve-year-old obese and non-obese children. Behav Res Ther 35:863-873.

Braet C, Soetens B, Moens E, Mels S, Goossens L, Van Vlierberghe L (2007) Are two informants better than one? Parent-child agreement on the eating styles of children who are overweight. Eur Eat Disord Rev 15:410-417.

Burton P, Smit HJ, Lightowler HJ (2007) The influence of restrained and external eating patterns on overeating. Appetite 49:191-197.

Canli T (2004) Functional brain mapping of extraversion and neuroticism: learning from individual differences in emotion processing. J Pers 72:1105-1132.

Coccaro EF, McCloskey MS, Fitzgerald DA, Phan KL (2007) Amygdala and orbitofrontal reactivity to social threat in individuals with impulsive aggression. Biol Psychiatry 62:168-178.

Cools R, Clark L, Owen AM, Robbins TW (2002) Defining the neural mechanisms of probabilistic reversal learning using event-related functional magnetic resonance imaging. J Neurosci 22:4563-4567.

Cornier MA, Von Kaenel SS, Bessesen DH, Tregellas JR (2007) Effects of overfeeding on the neuronal response to visual food cues. Am J Clin Nutr 86:965-971.

Davidson RJ, Putnam KM, Larson CL (2000) Dysfunction in the neural circuitry of emotion regulation-a possible prelude to violence. Science 289:591-594.

Delgado MR, Nearing KI, Ledoux JE, Phelps EA (2008) Neural circuitry underlying the regulation of conditioned fear and its relation to extinction. Neuron 59:829-838.
DelParigi A, Chen K, Salbe AD, Hill JO, Wing RR, Reiman EM, Tataranni PA (2007) Successful dieters have increased neural activity in cortical areas involved in the control of behavior. Int J Obes (Lond) 31:440-448.

Draganski B, Kherif F, Klöppel S, Cook PA, Alexander DC, Parker GJ, Deichmann R, Ashburner J, Frackowiak RS (2008) Evidence for segregated and integrative connectivity patterns in the human basal ganglia. J Neurosci 28:7143-7152.

Dresel C, Castrop F, Haslinger B, Wohlschlaeger AM, Hennenlotter A, Ceballos-Baumann AO (2005) The functional neuroanatomy of coordinated orofacial movements: sparse sampling fMRI of whistling. Neuroimage 28:588-597.

Egner T, Etkin A, Gale S, Hirsch J (2008) Dissociable neural systems resolve conflict from emotional versus nonemotional distracters. Cereb Cortex 18:1475-1484.

Ehrsson HH, Geyer S, Naito E (2003) Imagery of voluntary movement of fingers, toes, and tongue activates corresponding body-part-specific motor representations. J Neurophysiol 90:3304-3316.

Elfhag K, Tynelius P, Rasmussen F (2007) Sugar-sweetened and artificially sweetened soft drinks in association to restrained, external and emotional eating. Physiol Behav 91:191-195.

Elfhag K, Tholin S, Rasmussen F (2008) Consumption of fruit, vegetables, sweets and soft drinks are associated with psychological dimensions of eating behaviour in parents and their 12-year-old children. Public Health Nutr 11:914-923.

Etkin A, Egner T, Peraza DM, Kandel ER, Hirsch J (2006) Resolving emotional conflict: a role for the rostral anterior cingulate cortex in modulating activity in the amygdala. Neuron 51:871-882.

Fakra E, Salgado-Pineda P, Delaveau P, Hariri AR, Blin O (2008) Neural bases of different cognitive strategies for facial affect processing in schizophrenia. Schizophr Res 100:191-205.

Friedman DP, Aggleton JP, Saunders RC (2002) Comparison of hippocampal, amygdala, and perirhinal projections to the nucleus accumbens: combined anterograde and retrograde tracing study in the Macaque brain. J Comp Neurol 450:345-365.

Friedman MI, Ulrich P, Mattes RD (1999) A figurative measure of subjective hunger sensations. Appetite 32:395-404.

Friston KJ (1997) Testing for anatomically specified regional effects. Hum Brain Mapp 5:133-136.

Friston KJ, Holmes AP, Worsley KJ, Poline JB, Frith CD, Frackowiak RSJ (1994) Statistical parametric maps in functional imaging: a general linear approach. Hum Brain Mapp 2:189-210.

Friston KJ, Buechel C, Fink GR, Morris J, Rolls E, Dolan RJ (1997) Psychophysiological and modulatory interactions in neuroimaging. Neuroimage 6:218-229.

Ghashghaei HT, Hilgetag CC, Barbas H (2007) Sequence of information processing for emotions based on the anatomic dialogue between prefrontal cortex and amygdala. Neuroimage 34:905-923.

Gitelman DR, Penny WD, Ashburner J, Friston KJ (2003) Modeling regional and psychophysiologic interactions in fMRI: the importance of hemodynamic deconvolution. Neuroimage 19:200-207.

Goense JB, Logothetis NK (2008) Neurophysiology of the BOLD fMRI signal in awake monkeys. Curr Biol 18:631-640.

Haber SN, McFarland NR (1999) The concept of the ventral striatum in nonhuman primates. Ann N Y Acad Sci 877:33-48.

Haber SN, Kim KS, Mailly P, Calzavara R (2006) Reward-related cortical inputs define a large striatal region in primates that interface with associative cortical connections, providing a substrate for incentive-based learning. J Neurosci 26:8368-8376.

Hörchner R, Tuinebreijer W, Kelder H (2002) Eating patterns in morbidly obese patients before and after a gastric restrictive operation. Obes Surg 12:108-112.

Howell D (2007) Statistical methods for psychology, Ed 6. Florence, KY: Wadsworth.

Joel D (2001) Open interconnected model of basal ganglia-thalamocortical circuitry and its relevance to the clinical syndrome of Huntington's disease. Mov Disord 16:407-423.

Kelley AE, Baldo BA, Pratt WE, Will MJ (2005) Corticostriatalhypothalamic circuitry and food motivation: integration of energy, action and reward. Physiol Behav 86:773-795.

Killgore WD, Yurgelun-Todd DA (2006) Affect modulates appetite-related brain activity to images of food. Int J Eat Disord 39:357-363.

Killgore WD, Young AD, Femia LA, Bogorodzki P, Rogowska J, Yurgelun- 
Todd DA (2003) Cortical and limbic activation during viewing of highversus low-calorie foods. Neuroimage 19:1381-1394.

Knutson B, Bossaerts P (2007) Neural antecedents of financial decisions. J Neurosci 27:8174-8177.

Körner A, Kiess W, Stumvoll M, Kovacs P (2008) Polygenic contribution to obesity: genome-wide strategies reveal new targets. Front Horm Res $36: 12-36$.

Kumar A, Ajilore O (2008) Magnetic resonance imaging and late-life depression: potential biomarkers in the era of personalized medicine. Am J Psychiatry 165:166-168.

Kunishio K, Haber SN (1994) Primate cingulostriatal projection: limbic striatal versus sensorimotor striatal input. J Comp Neurol 350:337-356.

LaBar KS, Gitelman DR, Parrish TB, Kim YH, Nobre AC, Mesulam MM (2001) Hunger selectively modulates corticolimbic activation to food stimuli in humans. Behav Neurosci 115:493-500.

Li S, Loos RJ (2008) Progress in the genetics of common obesity: size matters. Curr Opin Lipidol 19:113-121.

Logothetis NK, Pauls J, Augath M, Trinath T, Oeltermann A (2001) Neurophysiological investigation of the basis of the fMRI signal. Nature 412:150-157.

Maldjian JA, Laurienti PJ, Kraft RA, Burdette JH (2003) An automated method for neuroanatomic and cytoarchitectonic atlas-based interrogation of fMRI data sets. Neuroimage 19:1233-1239.

Marsh AA, Finger EC, Mitchell DG, Reid ME, Sims C, Kosson DS, Towbin KE, Leibenluft E, Pine DS, Blair RJ (2008) Reduced amygdala response to fearful expressions in children and adolescents with callousunemotional traits and disruptive behavior disorders. Am J Psychiatry 165:712-720.

Masuda R, Fukuda M, Ono T, Endo S (1997) Neuronal responses at the sight of objects in monkey basal forebrain subregions during operant visual tasks. Neurobiol Learn Mem 67:181-196.

Monk CS, Telzer EH, Mogg K, Bradley BP, Mai X, Louro HM, Chen G, McClure-Tone EB, Ernst M, Pine DS (2008) Amygdala and ventrolateral prefrontal cortex activation to masked angry faces in children and adolescents with generalized anxiety disorder. Arch Gen Psychiatry 65:568-576.

Nishijo H, Ono T, Nishino H (1988) Topographic distribution of modalityspecific amygdalar neurons in alert monkey. J Neurosci 8:3556-3569.

Nishijo H, Yamamoto Y, Ono T, Uwano T, Yamashita J, Yamashima T (1997) Single neuron responses in the monkey anterior cingulate cortex during visual discrimination. Neurosci Lett 227:79-82.

Ochsner KN, Gross JJ (2005) The cognitive control of emotion. Trends Cogn Sci 9:242-249.

Ochsner KN, Ray RD, Cooper JC, Robertson ER, Chopra S, Gabrieli JD, Gross JJ (2004) For better or for worse: neural systems supporting the cognitive down- and up-regulation of negative emotion. Neuroimage 23:483-499.

O'Doherty JP (2004) Reward representations and reward-related learning in the human brain: insights from neuroimaging. Curr Opin Neurobiol 14:769-776.

O’Doherty JP, Buchanan TW, Seymour B, Dolan RJ (2006) Predictive neural coding of reward preference involves dissociable responses in human ventral midbrain and ventral striatum. Neuron 49:157-166.
Passamonti L, Rowe JB, Ewbank M, Hampshire A, Keane J, Calder AJ (2008) Connectivity from the ventral anterior cingulate to the amygdala is modulated by appetitive motivation in response to facial signals of aggression. Neuroimage 43:562-570.

Phelps EA, Delgado MR, Nearing KI, LeDoux JE (2004) Extinction learning in humans: role of the amygdala and vmPFC. Neuron 43:897-905.

Ploghaus A, Becerra L, Borras C, Borsook D (2003) Neural circuitry underlying pain modulation: expectation, hypnosis, placebo. Trends Cogn Sci 7:197-200.

Rolls ET, Burton MJ, Mora F (1980) Neurophysiological analysis of brainstimulation reward in the monkey. Brain Res 194:339-357.

Russchen FT, Bakst I, Amaral DG, Price JL (1985) The amygdalostriatal projections in the monkey. An anterograde tracing study. Brain Res 329:241-257.

Sawyer-Radloff L (1977) The CES-D scale: a self-report depression scale for research in the general population. Appl Psychol Meas 1:385-401.

Schiltz CA, Bremer QZ, Landry CF, Kelley AE (2007) Food-associated cues alter forebrain functional connectivity as assessed with immediate early gene and proenkephalin expression. BMC Biol 5:16.

Small DM (2002) Toward an understanding of the brain substrates of reward in humans. Neuron 33:668-671.

Small DM, Bender G, Veldhuizen MG, Rudenga K, Nachtigal D, Felsted J (2007) The role of the human orbitofrontal cortex in taste and flavor processing. Ann N Y Acad Sci 1121:136-151.

Spielberger C, Gorsuch R, Lushene P, Vagg P, Jacobs A (1983) Manual for the state-trait anxiety inventory (Form Y). Palo Alto, CA: Consulting Psychologists.

Tzourio-Mazoyer N, Landeau B, Papathanassiou D, Crivello F, Etard O, Delcroix N, Mazoyer B, Joliot M (2002) Automated anatomical labeling of activations in SPM using a macroscopic anatomical parcellation of the MNI MRI single-subject brain. Neuroimage 15:273-289.

van Strien T, Frijters JER, Bergers GPA, Defares PB (1986) The Dutch Eating Behaviour Questionnaire (DEBQ) for assessment of restrained, emotional, and external eating behavior. Int J Eat Disord 5:295-315.

Wang GJ, Volkow ND, Thanos PK, Fowler JS (2004a) Similarity between obesity and drug addiction as assessed by neurofunctional imaging: a concept review. J Addict Dis 23:39-53.

Wang GJ, Volkow ND, Telang F, Jayne M, Ma J, Rao M, Zhu W, Wong CT, Pappas NR, Geliebter A, Fowler JS (2004b) Exposure to appetitive food stimuli markedly activates the human brain. Neuroimage 21:1790-1797.

Wang GJ, Yang J, Volkow ND, Telang F, Ma Y, Zhu W, Wong CT, Tomasi D, Thanos PK, Fowler JS (2006) Gastric stimulation in obese subjects activates the hippocampus and other regions involved in brain reward circuitry. Proc Natl Acad Sci U S A 103:15641-15645.

Whitwell JL, Sampson EL, Loy CT, Warren JE, Rossor MN, Fox NC, Warren JD (2007) VBM signatures of abnormal eating behaviours in frontotemporal lobar degeneration. Neuroimage 35:207-213.

Will MJ, Franzblau EB, Kelley AE (2004) The amygdala is critical for opioidmediated binge eating of fat. Neuroreport 15:1857-1860.

Worsley KJ, Marrett S, Neelin P, Vandal AC, Friston KJ, Evans AC (1996) A unified statistical approach for determining significant signals in images of cerebral activation. Hum Brain Mapp 4:58-73. 